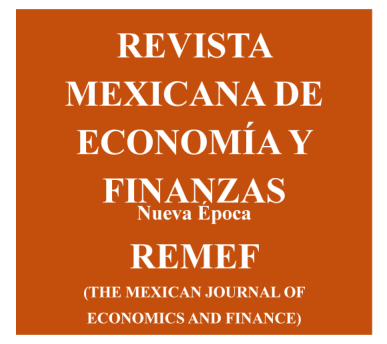
Revista Mexicana de Economía y Finanzas Nueva Época

Volumen 15 Número 3, Julio - Septiembre 2020, pp.395.413

DOI: https://doi.org/10.21919/remef .v15i3.465

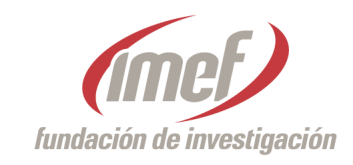

(Recibido: 6/enero/2020, aceptado: 2/junio/2020)

\title{
Federalismo fiscal y las asignaciones de transferencias en San Luis Potosí, México
}

\author{
Pedro Isidoro González Ramírez ${ }^{1}$ \\ Universidad Autónoma de San Luis Potosí, México \\ Edgar Gómez Galarza ${ }^{2}$ \\ Universidad Autónoma de San Luis Potosí, México
}

\section{Resumen}

El objetivo es estudiar la asignación de transferencias que realiza el Gobierno Federal, bajo el marco del federalismo fiscal, a los 58 municipios del estado de San Luis Potosí durante el periodo 2000-2018. Con un panel de datos de 1102 observaciones se estimó un modelo de regresión múltiple para datos agrupados y un modelo de efectos fijos. Las transferencias estudiadas fueron las del Ramo 33, del Fondo General de Participaciones y del Fondo de Fomento Municipal. Los resultados muestran que en las del Ramo 33 y del Fondo de Fomento Municipal no se observa influencia de filiación política como determinante para la asignación de recursos. Mientras que en las del Fondo General de Participaciones existe evidencia estadísticamente significativa de mayores asignaciones de recursos para aquellos municipios con filiación política tanto federal como conjunta. El estudio puede replicarse en otros municipios para obtener mayor evidencia. La propuesta es original para el estudio de transferencias hacia los municipios de San Luis Potosí. Se concluye que la filiación política es un factor que determina una mayor asignación de transferencias hacia municipios en el periodo estudiado. Clasificación JEL: C01,E62, Hry

Palabras claves: Federalismo Fiscal, Asignación de transferencias, filiación política

\section{Fiscal Federalism and transfer allocations in San Luis Potosí, Mexico}

\section{Abstract}

The aim is to study the assignment of transfers to the 58 municipalities of the State of San Luis Potosi during the period 2000-2018. With a panel data of 1102 observations, a pooled regression and fixed effect model was estimated. We studied the allocation of resources from Ramo 33, of the Fondo General de Participaciones and the Fondo de Fomento Municipal. The results of this study indicated for both Ramo 33 and Fondo de Fomento Municipal, that there is no influence of policy alignment as a determinant for resource allocation. However, for the Fondo General de Participaciones there is a statistically significant evidence of increased resource allocations for those municipalities with both federal and joint political alienation. This study can be replicated in other municipalities to obtain more evidence. The proposal is original for the study of transfers to San Luis Potosí municipalities. It is concluded that political alienation is a factor that determines more transfers allocations to municipalities in the studied period.

JEL Classification: C01,E62, Hr7

Keywords: Fiscal Federalism, transfers, political allegiance

${ }_{1}^{1}$ pedro.gonzalez@uaslp.mx. Av. Pintores s/n Col. Burócratas del Estado, C.P. 78213, San Luis Potosí, S.L.P., México.
${ }_{2}^{2}$ edgar.gomezgal@hotmail.com Av. Pintores s/n Col. Burócratas del Estado, C.P. 78213, San Luis Potosí, S.L.P., México. 


\section{Introducción}

La economía de México se encuentra fuertemente centralizada. A pesar de ser una república federal en la cual el país se divide en estados y municipios, los ingresos de estos dos niveles de gobierno dependen fuertemente de la hacienda federal. En este sentido, Sobarzo (1999) menciona que en México las relaciones intergubernamentales se han caracterizado por una fuerte centralización de funciones tanto de ingreso como de gasto en el ámbito federal del gobierno. Este poder central se encargó de controlar al país en todo lo que implicara grandes decisiones. La recaudación de impuestos no fue la excepción, ya que, como lo menciona Sobarzo (2006), el gobierno federal no sólo monopolizó el gasto público, también hizo lo propio con la recaudación de impuestos.

México cuenta en la actualidad con El Sistema Nacional de Coordinación Fiscal (SNCF), que tiene su origen en el año de 1980 cuando se creó el impuesto al valor agregado (IVA). Con la implementación de este sistema, se generaron atribuciones para que el gobierno federal concentrara la recaudación impositiva, restando capacidad recaudatoria a los estados y municipios. Dichas atribuciones quedaron claras en el marco legal planteado; sin embargo, como hace notar Sobarzo (1999), en el caso del gasto público, la distribución de competencias por ámbitos de gobierno ha sido mucho más discrecional y poco sistemática, caracterizada por la falta de reglas de asignación.

El gobierno federal, mediante el SNCF, se encargó de cobrar una serie de impuestos con los que se creó un fondo, el cual se repartiría a los gobiernos estatales y estos a su vez, a los municipios. De este modo, el gobierno federal comenzó a realizar transferencias hacia los estados y los municipios para que pudieran cumplir con sus obligaciones como prestadores de servicios públicos. Arroyo y Sánchez (1996) coinciden con este planteamiento al considerar que el SNCF armonizó el sistema tributario entre los estados de la Federación.

Downs (1957) considera que los gobiernos actúan de manera racional, como es descrito en la teoría económica, buscando maximizar sus beneficios o su utilidad, la cual depende del apoyo político (votos). Por lo tanto, llevan a cabo un mayor gasto hacia aquellas áreas en las cuales se obtengan mayores votos, y dejan de lado a las que no generen tantos.

Lo anterior es relevante al tomar en consideración lo planteado por Oates (2005), respecto a la segunda generación de la teoría del federalismo fiscal. El autor determina que la existencia de dependencia fiscal en los órdenes locales incentiva la disminución de ingresos propios. Del mismo modo, plantea, contrario a lo que se desarrolla en la primera generación de la teoría del federalismo fiscal, que los funcionarios públicos no necesariamente toman decisiones buscando el bien común.

El Estado entonces es visto como una entidad que basa sus decisiones en preferencias e intereses propios. La asignación de recursos se convierte en un mecanismo a través del cual el gobierno nacional puede influir en el comportamiento de los gobiernos municipales. El aumento o la disminución de transferencias condiciona el actuar de las unidades subnacionales. El Gobierno Federal tendría incentivos para apoyar a aquellos estados o municipios que sean gobernados por su mismo partido político o coalición.

En países que cuentan con un régimen fiscal con alto grado de centralización, los bienes públicos locales se administran de manera discrecional (Weingast, 2013). Esto da como resultado que todo aquello que el Estado debería brindar para la población en general, se brinda sólo a una parte de la población con la finalidad de influir en su comportamiento en los procesos electorales.

Diversos estudios han analizado el papel de las transferencias que realizan los gobiernos federales hacia los municipios. Sour (2013), establece que, al aumentar el monto de estas participaciones, los gobiernos municipales gastan más que el equivalente a un aumento en el ingreso privado. ${ }^{3}$

Stein (1999) por su parte, considera que, bajo determinadas circunstancias, el federalismo fiscal genera que los gobiernos aumenten su gasto; debido, precisamente, a que los ingresos no son recaudados, sino otorgados

\footnotetext{
${ }^{3}$ En la literatura este efecto se conoce como efecto "flypaper".
} 
por la federación. Este gasto excesivo suele destinarse a cuestiones de gasto corriente y no a actividades productivas. Pérez (2002) encontró que los ingresos vía participaciones federales representan la mayoría de los ingresos en los municipios de México, esta cifra es cercana al 60

El componente político puede influir en las decisiones tomadas tanto en la administración pública federal como en la estatal. En este sentido, Gámez e Ibarra-Yúnez (2009) señalan como posible línea de investigación el estudio de gobiernos estatales con mayoría en el Congreso respecto a los que no la tienen. Afirman que se podría esperar que los gobernadores que cuenten con mayoría en el Congreso tuvieran una conducta más oportunista. Reyes, Mejía y Riguzzi (2013) estudian los efectos que en las decisiones presupuestales tiene el hecho de que el presidente de la República tenga la misma filiación política que la mayoría de los diputados.

Valenzuela e Hinojosa (2017) desarrollan en su trabajo un modelo de regresión para un panel de datos con información de las 31 entidades federativas mexicanas para el período 2005-2014, donde incorporan la variable contrapeso político como variable dicotómica, que asume valores de 1 cuando el gobernador y la mayoría de los legisladores del congreso local de la entidad i en el año t provienen del mismo partido político; y valor de 0 en caso contrario. Los autores encuentran efectos positivos en el nivel de ingresos provenientes de fuentes tributarias cuando la mayoría de los legisladores locales tiene una filiación partidista distinta a la del gobernador. Sin embargo, su estudio se enfoca en determinar los efectos que las transferencias federales tienen en el desempeño fiscal local.

Kraemer (1997), citado en Valenzuela e Hinojosa (2017), analiza los sistemas de transferencias en Argentina, México y Brasil, y concluye tres aspectos fundamentales: i) no se observa principio de equidad, pues los estados con mayores ingresos per cápita recibieron mayores transferencias per cápita; ii) no funciona el principio de incentivo, dado que el esfuerzo fiscal de los estados no afecta la distribución de transferencias; y iii) se viola el principio de no discriminación política, ya que en el sistema de transferencias las decisiones sobre ingresos, gasto y deuda son asumidas bajo criterios políticos.

Ruiz-Porras y García-Vázquez (2014) consideran que el partido político que gobierna en el nivel federal tiene una disposición mayor para aumentar las participaciones hacia los municipios en los cuales gobierna el mismo partido. Llegaron a esta conclusión examinando las tendencias cuantitativas sobre las transferencias planeadas para grupos de municipios con niveles de desarrollo y de preferencia política comunes, tomando como base un panel de datos de 873 observaciones en los 125 municipios del estado de Jalisco en el periodo 2005-2011.

Esto también puede presentarse en los niveles locales. Ibarra, González y Sotres (2013) analizaron la filiación política entre los municipios y los gobiernos estatales. Cuando el alcalde y el gobernador son del mismo partido político, existe una mayor dependencia financiera entre ambos. También cuando los municipios son gobernados por un partido político que mayoritariamente controla el congreso, pueden surgir situaciones de endeudamiento significativas. Todo ello condiciona la salud de las finanzas públicas en ese nivel de gobierno.

La idea central de esta investigación es analizar si para el estado de San Luis Potosí bajo el marco del federalismo fiscal en México, el Gobierno Federal tiende a otorgar un mayor monto de recursos a aquellos municipios del estado con los que comparte filiación política. También se analiza si el otorgamiento de recursos se da cuando el partido político a nivel estatal comparte filiación política federal, si es así, se genera una filiación política entre los tres órdenes de gobierno. ${ }^{4} \mathrm{Si}$ bien existen estudios que analizan esta problemática, no se encontraron estudios para el estado de San Luis Potosí, por lo que la presente investigación pretende ayudar a la generación de evidencia sobre el federalismo fiscal y las aportaciones hacia los municipios potosinos.

Las asignaciones de recursos que fueron estudiadas fueron el Ramo general 33, el Fondo General de Participaciones y el Fondo de Fomento Municipal, del 2000 al 2018. Con los datos agrupados se generó un panel de 1102 observaciones. Para estos datos se generaron modelos de estimación, los cuales se han analizado

\footnotetext{
${ }^{4}$ Esto se ha denominado filiación conjunta.
} 
a través de la regresión múltiple y con el modelo de efectos fijos. Las estimaciones se realizaron para cada uno de los fondos mencionados.

Los resultados muestran que, tanto para el Ramo 33 como el Fondo de Fomento Municipal, no se observa influencia de filiación política federal o conjunta en los distintos órdenes de gobierno como determinantes para la asignación de recursos; sin embargo, para el Fondo General de Participaciones, sí se observa evidencia estadística significativa de mayores asignaciones de recursos per cápita para aquellos municipios con filiación política tanto federal como conjunta.

La centralización de las principales fuentes de recaudación y el reparto de los recursos que de ahí se obtienen pueden generar problemas de ineficiencia en la asignación de recursos. Esta investigación presenta evidencia de que en el estado de San Luis Potosí dicha asignación para ciertos fondos puede responder a criterios de filiación política, por lo que no se estarían cumpliendo los objetivos planteados para cada fondo. De ser así, esta investigación aporta evidencia para el diseño y generación de mecanismos de reparto que definan claramente los principios económicos y las fórmulas técnicas que eliminen la posibilidad discrecional de criterios políticos.

El artículo se integra de la siguiente forma: en la siguiente sección, se revisa la literatura sobre el Sistema de Coordinación Fiscal en México y de cada uno de los fondos utilizados; posteriormente, se describe la metodología utilizada; se presentan los resultados y análisis de la investigación; finalmente, se presentan las conclusiones y recomendaciones.

\section{Revisión de la literatura}

Diversos estudios muestran los posibles efectos que pueden tener las transferencias federales a los estados o municipios. López y Mayo (2015) elaboran un análisis comparativo entre los estados mexicanos de Chiapas y Nuevo León en un periodo de 20 años, entre 1990 y 2010. Dicho trabajo concluye que el sistema de aportaciones federales llevado a cabo en México, a partir de la década de 1980, ha generado problemas de dependencia por parte de las economías locales, ya que gran parte de sus recursos son proporcionados por el gobierno federal.

Bueno, Netzahuatl y Sánchez (2017) analizaron las finanzas públicas municipales desde 1990 hasta el año 2010 y agruparon a cada uno de los municipios mexicanos, según el índice de marginación utilizado por el Consejo Nacional de Evaluación de Políticas de Desarrollo Social (CONEVAL); por un lado, encontraron que los municipios con más alta marginación tienen mayores problemas para tener ingresos propios, por lo que dependen en gran medida de las participaciones federales. Por otro lado, los municipios con menor grado de marginación dependen menos de las participaciones federales, pero recurren con frecuencia al endeudamiento público. Con esto se puede inferir que sus ingresos también son insuficientes para los servicios que deben brindar. De igual manera, analizaron otros factores para la asignación de participaciones federales como la filiación política de los gobiernos municipales, así como la presencia de los ciclos electorales. Aunque en el caso de ambos, no se encontró evidencia estadísticamente significativa en los modelos utilizados por los autores.

Iregui, Melo y Ramos (2004) estudian mediante datos tipo panel la recaudación del impuesto predial en Colombia en el período 1990-2002. Los autores encontraron una relación negativa entre las transferencias federales y la recaudación de dicho impuesto local.

Mendoza (2009) estimó, para el periodo 2003-2013, un modelo de vectores autorregresivos de datos panel para las 32 entidades en México. Concluye que existe mayor pereza recaudatoria, así como menor transparencia cuando se presentan mayores transferencias por parte del gobierno federal hacia las entidades.

Ibarra (2013) empleó un panel de datos para analizar cuatro diferentes variables en el periodo 1998-2006, con la información de corte transversal de los 31 estados que en aquel entonces formaban parte de la federa- 
ción. Su hallazgo fue encontrar evidencia estadística significativa de que las diferencias institucionales (leyes, reglamentos, etc.) ayudan a explicar los distintos grados de dependencia entre los estados y la federación. Bajo uno de los modelos planteados por el autor, se comprobó que la filiación política influye en la asignación de las participaciones federales.

Valenzuela e Hinojosa (2017) desarrollan en su trabajo un modelo de regresión para un panel de datos con información de las 31 entidades federativas mexicanas para el período 2005-2014, donde incorporan la variable contrapeso político como variable dicotómica, que asume valores de 1 cuando el gobernador y la mayoría de los legisladores del congreso local de la entidad i en el año t provienen del mismo partido político; y valor de 0 en caso contrario. Los autores encuentran efectos positivos en el nivel de ingresos provenientes de fuentes tributarias cuando la mayoría de los legisladores locales tiene una filiación partidista distinta a la del gobernador. Sin embargo, su estudio se enfoca en determinar los efectos que, sobre el desempeño fiscal local, tienen las transferencias federales.

Es común que los partidos políticos que no tienen mayoría en determinado periodo y la población en general, consideren que los gobiernos en el poder utilicen la política económica para influir en la percepción de sus habitantes y logren convencerlos de votar por su mismo partido en los siguientes procesos electorales. El trabajo de Mejía, Reyes y Melquiades (2016) se enfoca en el ciclo político presupuestal en el estado de México, que tuvo el mayor número de habitantes durante el periodo 1980-2011. En él, encontraron que diversos componentes del gasto público se han manejado con objetivos electorales en diferentes episodios. Si bien son ciertos sus hallazgos, también consideraron que debe hacerse un análisis más extensivo en la materia.

Para el caso de América Latina, Acuña, Carrasco, Carrasco y Cazor (2017) generaron un estudio de las transferencias realizadas hacía 345 comunas en Chile. Sus resultados arrojaron discrecionalidad en algunas de las transferencias generadas por el gobierno nacional. Además, un monto mayor de transferencias hacia aquellas comunas que son gobernadas por la misma coalición política. El aumento de las transferencias también tiende a ser mayor en época de elecciones (Corvalan, Cox y Osorio, 2018).

La asignación de los recursos públicos con criterios políticos también puede realizarse en el rubro de la inversión. Livert, Gainza y Mogollón (2018) encontraron que, para un panel de datos de 1990 a 2013, la asignación de inversión en Chile se concentró en áreas donde los resultados de las elecciones, tanto nacionales como municipales, tuvieron resultados favorables para la coalición del gobierno. Esto lleva a que los gobiernos nacionales concentren sus políticas más importantes en los municipios que se encuentran alineados, convirtiéndolos en activos valiosos en términos políticos (Livert, Gainza y Acuña, 2019; Toro y Lara, 2019).

Para el caso de Brasil, Soares y Melo (2016) realizaron un análisis descriptivo y una regresión estadística respecto a las transferencias voluntarias del Gobierno Brasileño generadas hacia los municipios entre 1995 y 2010. Los resultados indican que dichas transferencias se realizaron apegadas a criterios políticos y no a un carácter redistributivo.

Por su parte, Deda y Kauchakje (2019) analizaron la influencia de los distritos electorales de Brasil y cómo su desproporcionalidad genera incentivos para gastos y transferencias discrecionales hacia los mismos. Lo anterior da como resultado que existan diversos mecanismos para mantener la discrecionalidad en la asignación de recursos.

\section{1. $\quad$ El Sistema de Coordinación Fiscal en México}

Salazar, Musi y Cervantes (2001) mencionan que "el Sistema Nacional de Coordinación Fiscal comprende las relaciones fiscales de la federación con las entidades federativas y los municipios, las reglas de coordinación administrativa entre las diferentes autoridades fiscales, así como los organismos en materia de coordinación fiscal". 
"El sistema se creó para evitar los problemas generados por la doble tributación potencial que permite la Constitución federal" (Flamand, 2006). Todo esto llevó a que, a partir de la década de 1980, los estados de México perdieran su capacidad de generar recursos de manera propia y que el Gobierno Federal recaudara los principales impuestos para después establecer participaciones que repartiría a los estados y municipios.

De acuerdo con Aguilar (1996), el Sistema de Coordinación Fiscal cuenta con una serie de inconvenientes, entre los cuales destacan: a) la fuerte dependencia que los estados y municipios tienen de las participaciones federales; b) las fallas en la forma como se distribuyen las participaciones; c) el recurrente déficit operacional de los órdenes de gobierno estatal y municipal; e) la reducción de los esfuerzos fiscales de los estados y municipios en la recaudación de impuestos.

De los inconvenientes mencionados, el segundo es el que tiene relevancia para nuestro objeto de estudio, ya que se plantea que la distribución y asignación de recursos por parte del gobierno federal se puede realizar con criterios de filiación política.

\subsection{Transferencias no condicionadas}

Para Trujillo (2008), los aportes no condicionados "son aquellos de tipo general, es decir, de libre asignación por parte del receptor, cuya justificación reside en cuestiones de equidad. Son transferencias de nivelación o igualación, dirigidas a las jurisdicciones más pobres".

Hernández-Trillo y Jarillo (2007) hacen un especial énfasis en la clasificación de los distintos tipos de transferencias. Dentro de las transferencias no condicionadas, establecen que la razón de ser de dichas transferencias radica en el resarcir a las entidades por el hecho de renunciar a la recolección de impuestos debido a la adhesión dentro del pacto federal.

En el panel de datos donde se analizarán las finanzas públicas de los 58 municipios del estado de San Luis Potosí para el periodo 2000-2018, se toman en cuenta un par de rubros que entran en la categoría de transferencias no condicionadas. Por un lado, el Fondo General de Participaciones y por otro lado el Fondo de Fomento Municipal. El Fondo General de Participaciones se integra con $20 \%$ de la Recaudación Federal Participable (RFP), que obtenga la federación durante el ejercicio. De acuerdo con Amieva-Huerta (2010), la RFP "se compone de todos los impuestos federales: ISR. IVA, IEPS (excepto el porcentaje participable a los estados), impuestos al comercio exterior, accesorios y los derechos sobre la extracción de petróleo y minería, disminuidos con el total de las devoluciones por los mismos conceptos". Del 1\% de la RFP, el $16.8 \%$ se destina para la formación de un Fondo de Fomento Municipal. El 83.2\% restante incrementará dicho Fondo a las entidades que coordinen en materia de derechos (Amieva-Huerta, 2010).

De acuerdo con los proyectos de presupuestos de Egresos de la Federación, los recursos del Ramo General 28, donde está incluido el Fondo General de Participaciones y el Fondo de Fomento Municipal, no tienen un destino específico en el gasto de los gobiernos locales, así, su carácter principal es resarcitorio; por lo que, tiene como fin asignar los recursos de manera proporcional a la participación de las entidades en la actividad económica y la recaudación; por lo tanto, pretende generar incentivos para promover el crecimiento económico y el esfuerzo recaudatorio.

\subsection{Transferencias condicionadas}

Trujillo (2008), los aportes condicionados "son transferencias específicas que el gobierno central debe asignar para el financiamiento de programas que involucran beneficios a otras jurisdicciones". Ibarra (2003) menciona que "las aportaciones federales representan transferencias condicionadas hacia los estados y municipios para que sean aplicadas en educación básica, normal, tecnológica y de adultos, en servicios de salud, en infraestructura social y en seguridad pública". 
Sour (2007) es consistente con la idea de que "las transferencias condicionadas imponen al beneficiario una serie de restricciones para su ejercicio, como puede ser un destino específico", ya que los estados y los municipios tienen ya designadas las áreas a las cuales deben destinarse estos recursos.

En las participaciones condicionadas destacan los recursos del Ramo 33, también conocido como aportaciones federales a entidades federativas y municipios. Para esta investigación se utilizará el término de Ramo 33 .

Sour (2004) considera que un elemento importante en la conformación de los recursos del Ramo 33, en la actualidad, consiste en que "son recursos descentralizados y controlados por las legislaturas estatales y con las actividades establecidas en cada fondo por la federación, orientándose hacia actividades específicas de educación, salud, infraestructura básica seguridad pública y programas de alimentación y asistencia social". Cuando se establecen a cuáles rubros van destinados dichos recursos, se les conoce como recursos etiquetados, así, de acuerdo con Sour (2008), "La Ley de Coordinación Fiscal determina los porcentajes y las fórmulas mediante los cuales se asignan los recursos del Ramo 33 hacia los gobiernos locales. Dichas fórmulas se basan en criterios poblacionales, geográficos y socioeconómicos relativos a cada gobierno".

Ángeles-Castro, Salazar-Rivera y Sandoval-Contreras (2013) son congruentes con este planteamiento al establecer que, "originalmente, el ramo 33 incorporaba cinco fondos diferentes y actualmente incorpora siete fondos orientados a satisfacer las necesidades en las áreas de educación, salud, infraestructura social, seguridad pública, bienestar y el fortalecimiento de los municipios".

Mejía, (2012) define el Ramo 33 como aquel que "comprende las aportaciones federales para entidades federativas y municipios, además de incluir, múltiples áreas prioritarias para el desarrollo nacional". Díaz y Silva (2004) hablan de la importancia que ha tenido este ramo al señalar que "los principales bienes y servicios públicos en México son provistos por los gobiernos estatales y municipales dentro de un rubro de transferencias descentralizadas conocidas como transferencias del Ramo 33". Por su parte, Ruiz-Porras y García-Vázquez (2014) consideran que la asignación del Ramo 33 está sujeta a la ejecución de políticas sociales y su distribución depende primordialmente de la Recaudación Federal Participable y de los índices de pobreza.

Las trasferencias del Ramo 33 son consideradas en el análisis de este estudio, ya que al existir filiación entre el partido político que gobierna a nivel federal y a nivel municipal puede existir también una mayor asignación de recursos, independientemente de cuestiones poblacionales, de marginación y de pobreza dentro de cada municipio.

Como se mencionó en la problemática de la investigación, existen trabajos relacionados con el federalismo fiscal donde se analiza la asignación de recursos con criterio político. Díaz (1997) estudió la asignación de recursos a las 32 entidades del país. Años después (2004), comparó el federalismo de México con el de otros países de Latinoamérica. Por su parte, Hernández y Jarillo (2007) realizaron un estudio sobre uno de los fondos del Ramo 33, el Fondo de Infraestructura Social Municipal. Ibarra, González y Sotres (2013) establecieron la dependencia financiera de los municipios mexicanos debido a la confluencia política que se presenta entre los distintos órdenes de gobierno; mientras que Ruíz-Porras y García-Vázquez (2013, 2014) analizaron las transferencias hacia los municipios del estado de Jalisco. El trabajo de Ruíz Porras (2013) es la base metodológica para seleccionar las variables que se incluyen en el modelo de regresión; de esta forma se estimará la posible presencia de criterios políticos en las transferencias hacia los municipios del estado de San Luis Potosí. 


\section{Metodología}

Diversos trabajos de investigación en los últimos años han aplicado la metodología de datos tipo panel para mostrar los efectos que pueden tener las transferencias de la federación sobre los municipios o estados en diversos aspectos, por ejemplo: Iregui, Melo y Ramos (2004), Mendoza (2009), Ibarra (2013), Soares y Melo (2016), Valenzuela e Hinojosa (2017), Livert, Gainza y Mogollón (2018).

En concordancia con los mencionados autores, se considera que el uso de estimaciones tipo panel son las más adecuadas para capturar los efectos de filiación política en los municipios del estado de San Luis Potosí sobre la asignación de transferencias federales a los municipios. Adicionalmente, como menciona Baltagi (1995), los datos tipo panel tienen la ventaja de que, al combinar series de tiempo de las observaciones de corte transversal, proporcionan una mayor cantidad de datos, más variabilidad, menos colinealidad entre variables, más grados de libertad y una mayor eficiencia en las estimaciones. Además, reducen el sesgo posible si se agregan individuos o agentes en conjuntos numerosos. Permite enriquecer el análisis empírico de manera que no sería posible con sólo datos de corte transversal o de series de tiempo. Finalmente, no tomar en cuenta los efectos de cada entidad puede generar que los resultados se modifiquen al usar una u otra técnica. En conclusión, se considera que el método de datos tipo panel es el más adecuado para el objetivo de la presente investigación; sin embargo, se realiza también la estimación de datos agrupados para verificar si la relación se mantiene bajo otra estimación.

Para la construcción del panel de datos, se recopiló información del apartado de Finanzas Públicas Estatales y Municipales en la página web del Instituto Nacional de Estadística y Geografía (INEGI). Se obtuvo información de las finanzas públicas de los 58 municipios del estado de San Luis Potosí en el periodo 20002018. Específicamente, se recopiló información sobre las aportaciones federales del Ramo General 33, del Fondo General de Participaciones, del Fondo de Fomento Municipal y sobre los Ingresos propios recaudados por los municipios. Ésta última como variable de control para medir el desempeño fiscal de los municipios; igualmente, del INEGI se obtuvo información sobre el Índice Nacional de Precios al Consumidor para cada uno de los años.

En el Consejo Nacional de Población (CONAPO) se obtuvo información relativa a la población ${ }^{5}$ y al Índice de Marginación para cada uno de los municipios. Para efectos de análisis, se clasificaron los municipios según su densidad poblacional, como una variable proxy a su nivel de desarrollo local. Esta forma de medir el desarrollo local fue propuesta por Martínez, Flamand y Hernández (2008). Los municipios fueron clasificados en cinco grupos de densidad (medidos en hab/km2): muy baja densidad poblacional $(0<$ dens $<10)$, baja densidad (10 dens $<50)$, media (50 dens $<100)$, alta (100 dens $<1000)$ y muy alta densidad (dens 1000$)$.

Para conocer la filiación política de los gobiernos municipales, se obtuvo información del Consejo Estatal Electoral y Participación Ciudadana de San Luis Potosí (CEEPAC). Dicha información no fue de carácter numérico, sino sobre el partido político que gobernó a nivel municipal y se contrastó con el partido político que gobernó a nivel estatal y federal; si existió filiación política entre ambos órdenes de gobierno, se caracterizó en el panel de datos con el número 1; en caso de no existir coincidencia de partido político se caracterizó en el panel de datos con el número 0. Finalmente, dada la dependencia de los ingresos federales de los ingresos petroleros se incluyó como variable de control el precio de la mezcla mexicana de petróleo, obteniendo la información del Sistema de Información Energética (SIE) de la Secretaria de Energía.

El análisis de los 58 municipios en el periodo de 2000-2018 permitió construir un panel de datos de 1102 observaciones. Para una correcta interpretación, los datos fueron deflactados mediante el Índice Nacional de Precios al Consumidor $(2018=100)$. Adicionalmente, mediante los datos de población y sus tasas de

\footnotetext{
${ }^{5}$ Para el cálculo de población también se tomó en cuenta la tasa de crecimiento de población, ya que sólo se contaba con los datos poblacionales de los municipios para los años 2000, 2005, 2010 y 2015.
} 
crecimiento todos los valores fueron transformados a términos per cápita.

En el cuadro 1 se describen las variables que se emplean en el estudio y que serán utilizadas en los modelos de estimación, para esto se utiliza un panel de datos con un modelo de regresión agrupada y el modelo de efectos fijos. En este estudio se utilizan como variables dependientes los datos correspondientes al Ramo general 33, el Fondo General de Participaciones y el Fondo de Fomento Municipal.

Cuadro 1. Descripción de las variables

\begin{tabular}{|c|c|}
\hline Variable & Definición \\
\hline \multicolumn{2}{|r|}{ Variables dependientes. } \\
\hline Ramo 33 & $\begin{array}{l}\text { Aportaciones federales del Ramo general } 33 \text { para entidades federativas } \\
\text { y municipios, medido en términos per cápita. }(2018=100)\end{array}$ \\
\hline FGP & $\begin{array}{l}\text { Aportaciones asignadas a los municipios del Fondo General de } \\
\text { Participaciones, medido en términos per cápita. }(2018=100)\end{array}$ \\
\hline FFM & $\begin{array}{l}\text { Participaciones asignadas del Fondo de Fomento Municipal, } \\
\text { medido en términos per cápita. }(2018=100)\end{array}$ \\
\hline \multicolumn{2}{|r|}{ Variables explicativas } \\
\hline Denpob & $\begin{array}{l}\text { Densidad poblacional del municipio en términos cualitativos: } \\
1=\text { Muy baja, } 2=\text { Baja, } 3=\text { Media, } 4=\text { Alta y } 5=\text { Muy alta. }\end{array}$ \\
\hline Ims & $\begin{array}{l}\text { Índice de marginación social para el municipio. La marginación } \\
\text { social se presenta en términos cualitativos: } \\
1=\text { Muy baja, } 2=\text { Baja, } 3=\text { Media, } 4=\text { Alta y } 5=\text { Muy alta. }\end{array}$ \\
\hline Fpf & $\begin{array}{l}\text { Filiación política entre el partido político que gobierna a nivel } \\
\text { federal y municipal. Esta es una variable dummy: } \\
0=\text { No existe filiación política y } 1 \text { = Existe filiación política. }\end{array}$ \\
\hline Fpc & $\begin{array}{l}\text { Filiación política entre los tres órdenes de gobierno. Esta es } \\
\text { una variable dummy: } 0=\text { No existe filiación política y } \\
1=\text { Existe filiación política. }\end{array}$ \\
\hline Ingp & $\begin{array}{l}\text { Ingresos propios recaudados por los municipios. Compuesto por la } \\
\text { suma de impuestos propios, derechos, aprovechamientos y } \\
\text { productos. Variable proxy para medir la capacidad administrativa } \\
\text { de los municipios en la recaudación fiscal. }\end{array}$ \\
\hline PMME & Precios promedio anuales en dólares de la Mezcla Mexicana de Petróleo. \\
\hline
\end{tabular}

El análisis empírico busca analizar si el Gobierno Federal tiende a otorgar un mayor monto de recursos a aquellos municipios del estado de San Luis Potosí con los que comparten filiación política. Sin embargo, a ciertos municipios podrían asignárseles cantidades mayores de recursos por cuestiones económicas o de operación del propio fondo. Tomando en cuenta lo anterior, en las estimaciones se agregaron variables de control. Específicamente, se incluyó el precio de la mezcla mexicana de petróleo debido a la dependencia petrolera de las finanzas públicas federales, lo cual puede ir en detrimento de las transferencias a los municipios.

Ruiz-Porras y García-Vázquez (2014) consideran que las participaciones y aportaciones federales se reparten sobre la base del esfuerzo recaudatorio local, el número de habitantes y el grado de marginalidad se toman como variables explicativas la densidad poblacional, el índice de marginación social e ingresos propios recaudados por los municipios. ${ }^{6}$

En la ecuación 1) se pretende analizar el efecto de la filiación política federal (apf) con las asignaciones a cada uno de los municipios. Por su parte, en la ecuación 2) se analizan los efectos de una filiación política conjunta, que se da cuando el partido político a nivel estatal comparte filiación política federal, lo que genera

\footnotetext{
${ }^{6}$ Compuesto por la suma de impuestos propios, derechos, aprovechamientos y productos. Variable proxy para medir la capacidad administrativa de los municipios en la recaudación fiscal.
} 
una filiación política entre los tres órdenes de gobierno. Esto se ha denominado, para la presente investigación, filiación política conjunta.

$$
\begin{aligned}
& \ln \left(y_{i t}\right)=\alpha_{i t}+\beta_{1} F p f_{i t}+\beta_{2} i m s_{i t}+\beta_{3} \text { denpob }_{i t}+\beta_{4} \ln \left(i n g p_{i t}\right)+\beta_{5} \ln \left(P M M E_{i t}\right)+u_{i t} \\
& \ln \left(y_{i t}\right)=\alpha_{i t}+\beta_{1} F p c_{i t}+\beta_{2} i m s_{i t}+\beta_{3} \text { denpob }_{i t}+\beta_{4} \ln \left(i n g p_{i t}\right)+\beta_{5} \ln \left(P M M E_{i t}\right)+u_{i t}
\end{aligned}
$$

Donde $\beta_{1}$ representa en las ecuaciones 1) y 2) respectivamente, el efecto que la filiación política de dos órdenes de gobierno federal-municipal o de tres federal-estatal-municipal tiene sobre las transferencias condicionadas (Ramo 33) o no condicionadas (FGP y FFM) a los municipios. Es importante destacar que cada ecuación se estima para cada uno de los fondos definidos como variables explicativas.

\section{Resultados}

En esta sección se presentan los resultados de estimación para los tres fondos estudiados, el análisis se divide en la existencia de filiación política federal (gobierno federal-municipal) y filiación política de los tres órdenes de gobierno.

\subsection{Filiación política federal}

Para saber cuál es la influencia que tiene la filiación política de los distintos órdenes de gobierno en la asignación de recursos, en primera instancia, se calculó una prueba t para diferencia de medias ${ }^{7}$ con el fin de observar si existe una diferencia significativa entre los municipios con el mismo partido político gobernante que el de la federación. Es importante destacar que el objetivo de la puebla t es sólo observar si antes de realizar cualquier estimación econométrica existen diferencias significativas entre las asignaciones federales a los municipios de acuerdo con su filiación política.

La tabla 1 muestra que en las transferencias provenientes del Fondo General de Participaciones sí se observa una diferencia positiva y significativa cuando existe filiación política federal. En las del Ramo 33 y del Fondo de Fomento Municipal no se observan diferencias estadísticamente significativas. Esto puede deberse a que las reglas de operación del Ramo 33, a diferencia del Fondo General de Participaciones, son más claras con un etiquetado más preciso en cómo se asignan las transferencias federales a los municipios, lo cual reduce la discrecionalidad de criterios políticos en su asignación.

\begin{tabular}{|c|c|c|c|c|}
\hline \multicolumn{5}{|c|}{ FILIACIÓN POLÍTICA FEDERAL } \\
\hline & SÍ & NO & & \\
\hline FONDO & Promedio de transferencias per cápita & Promedio de transferencias per cápita & $\begin{array}{l}\text { Diferencia de Medias (Dif): } \\
\text { Promedio (sí)-promedio (no) }\end{array}$ & P-valor \\
\hline Ramo 33 & 1555.197 & 1601.99 & -46.793 & 0.8875 \\
\hline FGP & 746.3347 & 720.8624 & $25.4723^{* *}$ & 0.0119 \\
\hline FFM & 241.4947 & 240.591 & 0.9037 & 0.4346 \\
\hline
\end{tabular}

Tabla 1. Resultados prueba t de municipios con filiación política federal vs. municipios sin filiación política

Fuente: Estimaciones propias.

Para observar con más detalle los efectos de la filiación federal y siguiendo lo propuesto por Bueno, Netzahuatl y Sánchez (2017), se estimaron nuevamente pruebas t, pero ahora los municipios se dividieron de acuerdo con su índice de marginación social. La tabla 2 muestra que en varios fondos sí se observan

\footnotetext{
${ }^{7}$ La Diferencia de Medias es igual promedio de transferencias per cápita de los municipios con filiación política menos las transferencias per cápita de los municipios sin filiación política.
} 
diferencias positivas y significativas en la asignación a los municipios para aquellos que comparten el mismo partido gobernante.

En el caso de las transferencias provenientes del Ramo 33 a aquellos municipios con un índice de marginación social tanto muy bajo como muy alto, sí se observan diferencias positivas y significativas en la asignación de recursos. Por su parte, las del Fondo General de Participación para todos los índices de marginación, excepto medio, se observan diferencias significativas. En las del Fondo de Fomento Municipal se observan diferencias positivas solamente para un índice de marginación social baja.

Tabla 2. Resultados prueba t de municipios con filiación política federal vs. municipios sin filiación política federal por índice de marginación social.

\begin{tabular}{|c|c|c|c|c|}
\hline \multicolumn{3}{|c|}{ FILIACIÓN POLÍTICA FEDERAL } & \multirow[b]{3}{*}{$\begin{array}{c}\text { Diferencia de Medias (Dif): } \\
\text { Promedio (sí) - promedio (no) }\end{array}$} & \multirow[b]{3}{*}{ P-valor } \\
\hline & Sí & NO & & \\
\hline FONDO & $\begin{array}{l}\text { Promedio de transferencias } \\
\text { per cápita }\end{array}$ & $\begin{array}{l}\text { Promedio de transferencias } \\
\text { per cápita }\end{array}$ & & \\
\hline \multicolumn{5}{|c|}{ Índice de Marginación Social (muy baja) } \\
\hline Ramo 33 & 744.1992 & 652.1127 & $92.0865^{* *}$ & 0.0313 \\
\hline FGP & 714.7364 & 646.1045 & $68.6319^{*}$ & 0.0575 \\
\hline FFM & 172.8392 & 207.81 & -34.9708 & 0.9011 \\
\hline \multicolumn{5}{|c|}{ Índice de Marginación Social (baja) } \\
\hline Ramo 33 & 988.7002 & 1067.436 & -78.7358 & 0.882 \\
\hline FGP & 765.6868 & 695.8432 & $69.8436^{* *}$ & 0.0221 \\
\hline FFM & 258.3289 & 204.9551 & $53.3738^{* * *}$ & 0.0013 \\
\hline \multicolumn{5}{|c|}{ Îndice de Marginación Social (media) } \\
\hline Ramo 33 & 1384.589 & 1374.538 & 10.051 & 0.4199 \\
\hline FGP & 745.5479 & 741.1589 & 4.389 & 0.4128 \\
\hline FFM & 247.3974 & 249.0225 & -1.6251 & 0.5714 \\
\hline \multicolumn{5}{|c|}{ Îndice de Marginación Social (alta) } \\
\hline Ramo 33 & 1770.263 & 1808.371 & -38.108 & 0.7644 \\
\hline FGP & 747.541 & 719.9727 & $27.5683^{*}$ & 0.0501 \\
\hline FFM & 239.8971 & 243.3826 & -3.4855 & 0.6654 \\
\hline \multicolumn{5}{|c|}{ Índice de Marginación Social (muy alta) } \\
\hline Ramo 33 & 2185.994 & 1973.274 & $212.72^{*}$ & 0.0903 \\
\hline FGP & 735.022 & 695.81 & $39.212^{*}$ & 0.0917 \\
\hline FFM & 249.4978 & 227.0688 & 22.429 & 0.1478 \\
\hline
\end{tabular}

Fuente: Estimaciones propias.

Si bien los resultados de la prueba t sólo permiten parcialmente no rechazar la hipótesis de que pueda existir influencia política en la asignación de transferencias federales a los municipios, la diferencia en asignaciones puede deberse a la no inclusión de controles que permitan distinguir claramente el peso de la filiación política. Dado lo anterior, se estima el modelo econométrico, incluyendo una serie de controles que permitían reducir el sesgo en la interpretación.

El análisis inicia con un modelo de regresión agrupado para un panel de datos de 1102 observaciones. En las estimaciones se incluyen como controles el índice de marginación social, densidad poblacional, precios del petróleo e ingresos propios recaudos por los municipios. ${ }^{8}$

Al igual que los resultados de Ibarra (2013), el modelo (1) muestra que los municipios con filiación política federal reciben del Fondo General de Participaciones $1.69 \%$ más de recursos per cápita ( $\mathrm{p}=0.01)$ que aquellos sin filiación política federal. Los modelos 2 y 3 muestran que no se observa una relación significativa en la

\footnotetext{
${ }^{8}$ En los datos reportados en cada uno de los modelos el número de observaciones no coincide con las 1102 del panel de datos, esto debido a que la estimación es logarítmica y que en varios periodos para algunos municipios la transferencia federal de dicho fondo fue nula, lo que elimina en la estimación la observación.
} 
filiación política federal para el Ramo 33 y para el Fondo de Fomento Municipal. Un resultado conocido y que se observa en todas las estimaciones, es la dependencia de las aportaciones federales de todos los fondos con los precios del petróleo.

Los resultados de esta prueba son similares a los obtenidos por Bueno, Netzahuatl y Sánchez (2017), pues se observan mayores asignaciones a los municipios con más alta marginación, lo cual puede deberse a la dependencia que tienen estos municipios de las participaciones federales o a la aplicación de un principio de equidad en la distribución de las transferencias.

Tabla 3. Resultados de las estimaciones de los modelos de regresión agrupada con filiación política federal.

\begin{tabular}{|c|c|c|c|}
\hline & (1) & (2) & (3) \\
\hline VARIABLES & ln_FGP & ln_FFM & ln_Ramo33 \\
\hline \multirow{2}{*}{ Fpf } & $0.0169^{* * *}$ & -0.0114 & -0.02351 \\
\hline & $(0.001050)$ & $(.0095189)$ & $(.0168119)$ \\
\hline \multirow{2}{*}{ Ims } & $.01854^{* * *}$ & $0.04929^{* * *}$ & $0.2959^{* * *}$ \\
\hline & $(.0058865)$ & $(.0053893)$ & $(.0093892)$ \\
\hline \multirow{2}{*}{ Denpob } & $-0.0133^{* *}$ & $-0.0270^{* * *}$ & -0.0063 \\
\hline & $(.0055154)$ & $(.0049743)$ & $(.0088162)$ \\
\hline \multirow{2}{*}{ ln_ingp } & $0.0307^{* * *}$ & $0.02608^{* * *}$ & 0.0077 \\
\hline & $(.0063484)$ & $(.0057593)$ & $(.0101282)$ \\
\hline \multirow{2}{*}{ ln_PMME } & $0.1525^{* * *}$ & $0.1685^{* * *}$ & $0.258^{* * *}$ \\
\hline & $(.0101533)$ & $(.0098001)$ & $(.0162194)$ \\
\hline \multirow{2}{*}{ Constante } & $5.819^{* * *}$ & $4.674^{* * *}$ & $5.269^{* * *}$ \\
\hline & $(.0553356)$ & $(.0539512)$ & $(.0877872)$ \\
\hline Observaciones & 1,054 & 988 & 1,065 \\
\hline R-Cuadrada & 0.229 & 0.310 & 0.534 \\
\hline
\end{tabular}

Fuente: Estimaciones propias.

Se realizaron nuevamente análisis econométricos para identificar si la filiación política federal incide en la asignación de recursos a cada uno de los municipios, pero ahora se controlaron las diferencias de cada municipio mediante el modelo de efectos fijos. Se observó otra vez que sólo en el Fondo General de Participaciones existe diferencia significativa en las asignaciones a los municipios con filiación política federal, el modelo (4) muestra que aquellos municipios donde gobierna el mismo partido que la federación reciben en términos per cápita $2.19 \%$ más de recursos.

Tabla 4. Resultados de las estimaciones de los modelos de efectos fijos con filiación política federal.

\begin{tabular}{|c|c|c|c|}
\hline & $(\mathbf{4})$ & $\mathbf{( 4 )}$ & $\mathbf{( 6 )}$ \\
\hline VARIABLES & $\mathbf{l n}$ FGP & ln_FFM & ln_Ramo33 \\
\hline \multirow{2}{*}{ Fpf } & $0.0219^{* *}$ & -0.0084 & 0.0109 \\
\cline { 2 - 4 } & $(.0106998)$ & $(.0093556)$ & $(.0133845)$ \\
\hline \multirow{2}{*}{ Ims } & $-0.0393^{* *}$ & $-0.02465^{* *}$ & 0.01072 \\
\cline { 2 - 4 } & $(.0140097)$ & $(.0120408)$ & $(.0174749)$ \\
\hline \multirow{2}{*}{ Denpop } & $0.0672^{* *}$ & 0.0329 & -0.02173 \\
\cline { 2 - 4 } & $(.0267919)$ & $(.0248141)$ & $(.0333489)$ \\
\hline \multirow{2}{*}{$\ln$ _ingp } & $0.0480^{* * *}$ & $0.0288^{* * *}$ & $0.0424^{* * *}$ \\
\cline { 2 - 4 } & $(.0110438)$ & $(.0097502)$ & $(.0136836)$ \\
\hline \multirow{2}{*}{$\ln$ _PMME } & $0.1335^{* * *}$ & $0.157^{* * *}$ & $0.1941^{* * *}$ \\
\cline { 2 - 4 } & $(.0103756)$ & $(.0094268)$ & $(.0129796)$ \\
\hline Constante & $5.81^{* * *}$ & $4.816^{* * *}$ & $6.353^{* * *}$ \\
\hline
\end{tabular}




\begin{tabular}{|c|c|c|c|}
\hline Prueba de Hausman & & & \\
\hline & 0.0000 & 0.0000 & 0.0000 \\
\hline & 0.0000 & 0.0000 & 0.0000 \\
\hline \multicolumn{4}{|l|}{$\mathrm{F}$ test $\mathrm{P}$-valor } \\
\hline Observaciones & 1,054 & 978 & 1,065 \\
\hline R-cuadrada & 0.262 & 0.3051 & 0.251 \\
\hline Número de Municipios & 58 & 58 & 58 \\
\hline
\end{tabular}

Fuente: Estimaciones propias.

Los resultados obtenidos muestran que las transferencias de ciertos fondos pueden estar condicionadas por criterios políticos, lo cual es congruente con los resultados obtenidos para el caso mexicano por Ibarra (2013), Mejía, Reyes y Melquiades (2016), y para América Latina por Soares y Melo (2016), Acuña, Carrasco, Carrasco y Cazor (2017) y Livert, Gainza y Mogollón (2018).

\subsection{Filiación política conjunta}

Para conocer cuál es la influencia que tiene la filiación política de los tres órdenes de gobierno en la asignación de recursos, se estimó en primera instancia una prueba t en la diferencia de medias para observar si existe una diferencia significativa entre los municipios con filiación política conjunta, tal como se realizó en la sección anterior.

La tabla 5 muestra nuevamente que, en las transferencias del Fondo General de Participaciones, sí se observa una diferencia positiva y significativa cuando existe filiación política entre los tres órdenes de gobierno. En las del Ramo 33 y el Fondo de Fomento Municipal, no se observan diferencias estadísticamente significativas.

Tabla 5. Resultados prueba t de municipios con filiación política conjunta vs. municipios sin filiación política conjunta.

\begin{tabular}{|c|c|c|c|c|}
\hline & $\begin{array}{l}\text { FILIACIÓN POLÍ } \\
\text { SÍ }\end{array}$ & $\begin{array}{l}\text { ICA CONJUNTA } \\
\text { NO }\end{array}$ & & \\
\hline FONDO & $\begin{array}{l}\text { Promedio de transferencias } \\
\text { per cápita }\end{array}$ & $\begin{array}{l}\text { Promedio de transferencias } \\
\text { per cápita }\end{array}$ & $\begin{array}{l}\text { Diferencia de Medias (Dif): } \\
\text { Promedio (si) - promedio (no) }\end{array}$ & P-v: \\
\hline Ramo 33 & 1521.676 & 1608.242 & -86.566 & 0.9 \\
\hline FGP & 752.6667 & 722.8894 & $29.7773^{* * *}$ & 0.0 \\
\hline FFM & 242.7156 & 240.2089 & 2.5067 & 0.3 \\
\hline
\end{tabular}

Las pruebas t se estimaron otra vez dividiendo a los municipios de acuerdo con su índice de marginación social. La tabla 6 muestra que al menos en algún índice de marginación todos los fondos presentan diferencias positivas y significativas en la asignación de recursos para aquellos municipios con filiación política en los tres órdenes de gobierno. Por ejemplo, en el FGP para un índice de marginación social alto los municipios que tienen filiación política conjunta reciben en promedio 30.89 pesos $(\mathrm{p}=0.05)$ más en términos per cápita que aquellos que no la tienen.

Un resultado destacable de la tabla 6, congruente con los resultados obtenidos por Kraemer (1997), es que cuando se observa una diferencia en media negativa, es decir, que los municipios con filiación política conjunta reciben menos recursos per cápita que su contraparte, en término estadísticos esa diferencia no es 
significativa. Lo cual parece indicar que se viola el principio de no discriminación política, ya que se observa que las transferencias son asumidas bajo criterios políticos.

Tabla 6. Resultados prueba t de municipios con filiación política conjunta vs. municipios sin filiación política conjunta por índice de marginación social.

\begin{tabular}{|c|c|c|c|c|}
\hline \multicolumn{3}{|c|}{ FILIACIÓN POLÍTICA CONJUNTA } & \multirow[b]{3}{*}{$\begin{array}{c}\text { Diferencia de Medias (Dif): } \\
\text { Promedio (si) - promedio (no) }\end{array}$} & \multirow[b]{3}{*}{ P-valor } \\
\hline & SÍ & NO & & \\
\hline FONDO & $\begin{array}{l}\text { Promedio de transferencias } \\
\text { per cápita }\end{array}$ & $\begin{array}{c}\text { Promedio de transferencias } \\
\text { per cápita }\end{array}$ & & \\
\hline \multicolumn{5}{|c|}{ Índice de Marginación Social (muy baja) } \\
\hline Ramo 33 & 749.2163 & 668.4411 & $80.7752^{*}$ & 0.055 \\
\hline FGP & 728.9863 & 651.1418 & $77.8445^{* *}$ & 0.0389 \\
\hline FFM & 204.8421 & 178.6 & 26.2421 & 0.172 \\
\hline \multicolumn{5}{|c|}{ Índice de Marginación Social (baja) } \\
\hline Ramo 33 & 1019.437 & 1020.431 & -0.994 & 0.506 \\
\hline FGP & 796.3234 & 697.534 & $98.7894^{* * *}$ & 0.0019 \\
\hline FFM & 265.9816 & 217.1356 & $48.846^{* * *}$ & 0.003 \\
\hline \multicolumn{5}{|c|}{ Îndice de Marginación Social (media) } \\
\hline Ramo 33 & 1312.806 & 1406.493 & -93.687 & 0.859 \\
\hline FGP & 752.1765 & 739.2479 & 12.9286 & 0.2751 \\
\hline FFM & 241.666 & 251.0992 & -9.4332 & 0.8318 \\
\hline \multicolumn{5}{|c|}{ Índice de Marginación Social (alta) } \\
\hline Ramo 33 & 1750.054 & 1811.148 & -61.094 & 0.8592 \\
\hline FGP & 752.8228 & 721.9321 & $30.8907^{* *}$ & 0.043 \\
\hline FFM & 242.8793 & 241.5836 & 1.2957 & 0.4412 \\
\hline \multicolumn{5}{|c|}{ Índice de Marginación Social (muy Alta) } \\
\hline Ramo 33 & 2128.82 & 2075.078 & 53.742 & 0.3751 \\
\hline FGP & 703.1046 & 725.0471 & -21.9425 & 0.6776 \\
\hline FFM & 238.8125 & 240.0839 & -1.2714 & 0.5223 \\
\hline
\end{tabular}

Fuente: Estimaciones propias.

Se realizaron análisis econométricos para identificar si la filiación política conjunta incide en la asignación de recursos a cada uno de los municipios. El análisis inicia con un modelo de regresión agrupado que incluye las mismas variables de control que la sección anterior.

El modelo (7) muestra que del Fondo General de Participaciones aquellos municipios con filiación política conjunta reciben significativamente $4.17 \%$ más de recursos per cápita $(\mathrm{p}=0.001)$ que aquellos sin filiación política conjunta, más del doble que cuando sólo existe filiación política federal. Los modelos 8 y 9 muestran que no se observa una relación significativa en la filiación política conjunta para el Ramo 33 y para el Fondo de Fomento Municipal.

En todas las estimaciones se observa una relación negativa y significativa entre las aportaciones federales y el precio del petróleo, esto indica que los ingresos federales dependen de las rentas petroleras.

Respecto al índice de marginación social, se observa una relación positiva y significativa en todos los fondos estudiados, lo cual indica que los municipios con más alta marginación reciben en términos per cápita mayores recursos. 
Tabla 7. Resultados de las estimaciones de los modelos de regresión agrupada con filiación política

\begin{tabular}{|c|c|c|c|}
\hline \multirow{2}{*}{ conjunta. } & $\mathbf{( 7 )}$ & $\mathbf{( 8 )}$ & $\mathbf{( 9 )}$ \\
\hline VARIABLES & $\mathbf{l n} \_$FGP & $\mathbf{l n} \_$FFM & ln_Ramo33 \\
\hline \multirow{2}{*}{ Fpc } & $0.04171^{* * *}$ & 0.00519 & -0.03108 \\
\cline { 2 - 4 } & $(.0112159)$ & $(.0101983)$ & $(.180223)$ \\
\hline \multirow{2}{*}{ Ims } & $0.01916^{* * *}$ & $0.04972^{* * *}$ & $0.2957^{* * *}$ \\
\cline { 2 - 4 } & $(.0058555)$ & $(.0053954)$ & $(.0093852)$ \\
\hline \multirow{2}{*}{ Denpob } & $-0.01267^{* *}$ & $-0.02724^{* * *}$ & -0.0069 \\
\cline { 2 - 4 } & $(.0054817)$ & $(.0049737)$ & $(.0088002)$ \\
\hline \multirow{2}{*}{$\ln$ _ingp } & $0.0300^{* * *}$ & $0.02551^{* * *}$ & 0.0079 \\
\cline { 2 - 4 } & $(.0063161)$ & $(.0057634)$ & $(.0101254)$ \\
\hline \multirow{2}{*}{ ln_PMME } & $0.1520^{* * *}$ & $0.1678^{* * *}$ & $0.2578^{* * *}$ \\
\cline { 2 - 4 } & $(.0100919)$ & $(.0097904)$ & $(.0161973)$ \\
\hline \multirow{2}{*}{ Constante } & $5.816^{* * *}$ & $4.6724^{* * *}$ & $5.2708^{* * *}$ \\
\cline { 2 - 4 } & $(.0550417)$ & $(.0540182)$ & $(.0877498)$ \\
\hline Observaciones & 1,054 & 978 & 1,065 \\
\hline R-Cuadrada & 0.237 & 0.309 & 0.535 \\
\hline
\end{tabular}

Finalmente, se realizó un modelo de panel de datos con efectos fijos incluyendo la filiación política conjunta. Se observa nuevamente que solo el Fondo General de Participaciones existe diferencia significativa en las asignaciones a los municipios con filiación política conjunta, el modelo (9) muestra que aquellos municipios donde se da filiación de los tres órdenes de gobierno reciben en términos per cápita $4.14 \%$ más de recursos $(\mathrm{p}=0.001)$. Los modelos 10 y 11 muestran que no se observa una relación significativa en la filiación política conjunta para el Ramo 33 y para el Fondo de Fomento Municipal.

Un resultado no consistente en las estimaciones es el signo que tiene el índice de marginación social, en las estimaciones con datos agrupados tanto con filiación política federal como conjunta la relación es positiva; pero cuando se realizan estimaciones con efectos fijos el sentido de la relación cambia. Lo anterior no permite concluir si las transferencias asignadas por cada uno de los fondos estudiados a los municipios de San Luis Potosí cumplen con el principio de equidad.

Tabla 8. Resultados de las estimaciones de los modelos de efectos fijos con filiación política conjunta.

\begin{tabular}{|c|c|c|c|}
\hline & (9) & (10) & (11) \\
\hline VARIABLES & ln_FGP & ln_FFM & ln_Ramo33 \\
\hline \multirow{2}{*}{ Fpc } & $0.04145^{* * *}$ & 0.007304 & -0.0174 \\
\hline & $(.0113108)$ & $(.0099476)$ & $(.0141876)$ \\
\hline \multirow{2}{*}{ Ims } & $-0.0406^{* * *}$ & $-0.02587 * *$ & 0.0131 \\
\hline & $(.0139409)$ & $(.0120411)$ & $(.0174587)$ \\
\hline \multirow{2}{*}{ Denpob } & $0.06174^{* *}$ & 0.0314107 & -0.0244 \\
\hline & $(.0267281)$ & $(.024872)$ & $(0.0329)$ \\
\hline \multirow{2}{*}{ ln_ingp } & $0.04471^{* * *}$ & $0.02707^{* * *}$ & $0.04559^{* * *}$ \\
\hline & $(.0110476)$ & $(.009803)$ & $(.0137467)$ \\
\hline \multirow{2}{*}{ ln_PMME } & $0.1343^{* * *}$ & $0.1570^{* * *}$ & $0.1946^{* * *}$ \\
\hline & $(.0103151)$ & $(0.009415)$ & $(.0129599)$ \\
\hline
\end{tabular}




\begin{tabular}{|c|c|c|c|}
\hline Constante & $5.839^{* * *}$ & $4.828^{* * *}$ & $6.328^{* * *}$ \\
\hline Prueba de Hausman & & & \\
P-Valor & 0.0000 & 0.0000 & 0.0000 \\
& 0.0000 & 0.0000 & 0.0000 \\
F test P-valor & & & \\
\hline Observaciones & 1,054 & 978 & 1,065 \\
\hline R-Cuadrada & 0.269 & 0.305 & 0.252 \\
\hline Número de Municipios & 58 & 58 & 58 \\
\hline
\end{tabular}

Fuente: Estimaciones propias.

Con los resultados obtenidos, se concluye que, si bien en las transferencias del Ramo 33 y del Fondo de Fomento Municipal no se observan de forma significativa criterios de asignación por filiación política, en el mismo sentido que concluye Kramer (1997), en el caso de las del Fondo General de Participaciones sí se viola el principio de no discriminación política, puesto que son asumidas bajo criterios políticos.

\section{Conclusiones}

En países que cuentan con un régimen fiscal con alto grado de centralización, la recaudación y el reparto de los recursos que de ahí se obtienen pueden generar problemas de ineficiencia en la asignación de recursos. Diversos estudios han demostrado que dicha asignación puede darse de manera discrecional por cuestiones políticas y no técnicas.

El objetivo central de esta investigación fue analizar si el Gobiernos Federal tiende a otorgar un mayor monto de recursos a aquellos municipios de San Luis Potosí con los que comparten filiación política tanto federal como de los tres órdenes de gobierno.

Después de haber analizado la asignación de recursos del Ramo general 33, del Fondo General de Participaciones y del Fondo de Fomento Municipal del 2000 al 2018, se concluye que, en las transferencias del Ramo 33 y del Fondo de Fomento Municipal, no se observa influencia de filiación política, tanto federal como conjunta, como determinantes para la asignación de recursos. Sin embargo, para el Fondo General de Participaciones, sí se observa evidencia estadísticamente significativa de mayores asignaciones de recursos per cápita para aquellos municipios con filiación política tanto federal como conjunta.

Es relevante mencionar que en la actualidad los gobiernos municipales son ampliamente dependientes de los recursos que les asigna el Gobierno Federal, por lo que una de las tareas pendientes es que los gobiernos municipales puedan contar con una mayor cantidad de recursos obtenidos de manera propia. Esto tenderá a disminuir las transferencias por parte del Gobierno Federal y con base en las estimaciones obtenidas no se presten a ser asignadas por criterios de filiación política.

Los resultados obtenidos aportan evidencia para diseñar mecanismos de reparto que definan claramente los principios económicos y las fórmulas técnicas que eliminen la posibilidad discrecional de criterios políticos.

En la literatura económica relacionada con el campo de finanzas públicas, también se encuentran los temas de ciclo político económico y ciclo económico presupuestal. Éstos pueden ser complementarios para futuras líneas de investigación, con las que podría examinarse si en los años electorales el monto de participaciones y aportaciones del gobierno federal hacia los municipios fue mayor que en los años que no son considerados electorales. 


\section{Referencias}

[1] Aguilar, L. F. (1996). El federalismo mexicano: Funcionamiento y tareas pendientes. Revista Mexicana De Sociología,58(3), 3-37. doi:10.2307/3540873

[2] Acuña, H., Carrasco, D., Carrasco, M. y Cazor, A. (2017). Transferencias gubernamentales discrecionales para la captura de votos: el caso de Chile. Economía y Política, 4(1), 37-96. Recuperado de: https://dialnet.unirioja.es/servlet/articulo?codigo $=6266400$

[3] Amieva-Huerta, J. (2010). Finanzas Públicas en México. (2da edición). México. Porrúa.

[4] Ángeles-Castro, G., Salazar-Rivera, M. P. y Sandoval-Contreras, L. (2013). Federalismo fiscal, el Ramo 33 y su efecto sobre el crecimiento y la distribución del ingreso: datos para México. Es economía, 8(37), 27-53. doi:10.29265/gypp.v28i1.543

[5] Arroyo, A. y Sánchez, A. (1996). Federalismo fiscal y condiciones de las finanzas públicas municipales. Revista Mexicana de Sociología, 58(3), 119-132. doi:10.2307/3540877.

[6] Baltagi, B. (1995). Econometric Analysis of Panel Data (Third edition). Nueva York: John Wiley and Sons.

[7] Bueno, L. E., Netzahuatl, M. F. y Sánchez, K. F. (2017). Dependencia financiera de las transferencias federales en los municipios de México. Global de Negocios, 5(4), 13-25. Recuperado de: http://www.theibfr.com/ARCHIVE/RGN-V5N4-2017.pdfpage=15

[8] CONAPO. Índice de marginación (carencias poblacionales) por localidad, municipio y entidad. Recuperado de: https://datos.gob.mx/busca/dataset/indice-de-marginacion-carencias-poblacionales-por-localidadmunicipio-y-entidad

[9] Corvalan, A., Cox, P. y Osorio, R. (2018). Indirect political budget cycles: Evidence from Chilean municipalities. Journal of Development Economics, 133, 1-14. doi: 10.1016/j.jdeveco.2018.01.001

[10] Deda, C. C. y Kauchakje, S. (2019). Determinantes das transferências fiscais para as unidades municipais: efeitos da representação desproporcional dos distritos eleitorais brasileiros. Revista de Administração Pública, 53(1), 150-172. doi:10.1590/0034-761220170266.

[11] Díaz, A. (1997). Asignación política de recursos en el federalismo mexicano: incentivos y limitaciones. Perfiles Latinoamericanos, 10, 35-73. Recuperado de: http://www.redalyc.org/pdf/115/11501003.pdf

[12] _ (2004). El federalismo y los límites políticos de la redistribución. Gestión y Política Pública, $13(3), 663-687$. Recuperado de: http://www.gestionypoliticapublica.cide.edu/num_anteriores/Vol.XIII_NoIII_2dosem/ Diaz-Cayeros.pdf

[13] Díaz, A. y Silva, S. (2004). Descentralización a escala municipal en México: la inversión en infraestructura social. CEPAL. Recuperado de: http://repositorio.cepal.org/bitstream/handle/11362/4935/S043145_es . pdf? sequence=1\&isAllowed=y

[14] Flamand, L. (2006). El juego de la distribución de recursos en un sistema federal. La influencia del gobierno dividido verticalmente en la asignación de fondos federales a los estados mexicanos. Política y gobierno, 13(2), 315-359. Recuperado de: http://www.politicaygobierno.cide.edu/index.php/pyg/article/view/284/194

[15] Gámez, C., e Ibarra-Yúnez, A. (2009). El ciclo político oportunista y el gasto de los estados mexicanos. Gestión y Política Pública, 18(1), 39-65.

[16] Hernández, F. y Jarillo, B. (2007). Transferencias condicionadas federales en países en desarrollo: el caso del FISM en México. Estudios Económicos, 22(002), 143-184.

[17] Ibarra, J. (2003). Dependencia financiera en las participaciones federales de los estados fronterizos mexicanos. Frontera norte, 15(29), 87-123.

[18] Ibarra, J. (2013). Entorno político y dependencia financiera de los estados mexicanos. Gestión y Política Pública, $22(1), 03-44$.

[19] Ibarra J., González, H. y Sotres, L. (2013). Aspectos políticos de la dependencia financiera en los municipios mexicanos. Revista Mexicana de Ciencias Políticas y Sociales, 58(217), 139-170. doi:10.1016/s0185-1918(13)72278-5 
[20] INEGI. Estadística de Finanzas Públicas Estatales y Municipales. Conjunto de datos: Finanzas Públicas Municipales. Recuperado de: https://www.inegi.org.mx/sistemas/olap/consulta/general_ver4/MDXQueryDatos . asp?\#Regreso\&c=11289

[21] Iregui, A. M., Melo, L. y Ramos, J. (2005). El impuesto predial en Colombia: factores explicativos del recaudo. Revista de Economía del Rosario, 8(1), 25-58. doi:10.32468/be.319

[22] Kraemer, M. (1997). Transferencias entre gobiernos y representación política: elementos empíricos de juicio de Argentina, Brasil y México (No. 4069). Inter-American Development Bank, Research Department.

[23] Livert, F., Gainza, X. y Mogollón, R. (2018). Factores Políticos en la Distribución de Inversión Pública: Problemas de Planificación y Gobernanza en Chile. Recuperado de: https://www.sociedadpoliticaspublicas.cl/archivos/noveno/Politica_Livert_Felipe.pdf

[24] López, J. y Mayo, B. (2015). Federalismo fiscal. Chiapas y Nuevo León: un análisis comparativo. Economía UNAM, 12(34), 106-123. doi:10.1016/s1665-952x(15)30007-4

[25] Martínez, S., Flamand, L. y Hernández, A. (2008) Panorama del desarrollo municipal en México: Antecedentes, diseño y hallazgos del índice del desarrollo municipal básico, Gestión y Política Pública, 17(1), pp. 145-192

[26] Mejía, F. (2012). Presupuesto federal en educación y su distribución por tipo de gasto, ramo y fondo. Impacto de la fórmula de la distribución del FAEB, 2008-2012. Revista Latinoamericana de Estudios Educativos (México), 42(3), 21-34. Recuperado de: http://www.redalyc.org/html/270/27024686002/

[27] Mejía, P., Reyes, M. R. y Melquiades B. I. (2016). Evidencia del ciclo político presupuestal en el Estado de México. Economía: Teoría y Práctica, 45, 207-234. Recuperado de: http://www.izt.uam.mx/economiatyp/ojs

[28] Mendoza, A. (2019). ¿Pueden las transferencias federales afectar el esfuerzo fiscal, la transparencia y la rendición de cuentas de los gobiernos locales? México 2003-2013. El Trimestre Económico, 86(342), 307-342. doi:10.20430/ete.v86i342.747

[29] Oates, W. (2005). Towards a second-generation theory of fiscal federalism. International and Public Finance, 12(4), 349-373.

[30] Pérez, C. (2002). Finanzas públicas en los tres niveles de gobierno en México, $1990-1999$. Los retos de federalismo fiscal. Revista Momento Económico, 120, 35-53. Recuperado de: http://revistas.unam.mx/index.php/rme/article/view/4303/3836

[31] Reyes-Hernández, M. R., Mejía-Reyes, P. y Riguzzi, P. (2013). Ciclo político presupuestal y gobiernos con y sin mayoría en México, 1994 y 2006. Economía, sociedad y territorio, 13(41), 79-119. doi:10.22136/est00201319

[32] Ruíz-Porras, A., y García-Vázquez, N. (2013). La reforma hacendaria y las transferencias en los municipios de Jalisco 2005-2011. Economía Informa, 381, 29-40. doi:10.1016/s0185-0849(13)71326-4

[33] _ (2014). El federalismo fiscal y las transferencias planeadas hacia los municipios mexicanos: criterios económicos y políticos. Espiral (Guadalajara), 21(59), 69-86. Recuperado de: http://www.scielo.org.mx/pdf/espiral/v21n59/v21n59a3.pdf

[34] Salazar, J. I., Musi, A. S. y Cervantes, L. S. (2001). Participaciones federales y dependencia de los gobiernos municipales en México, 1975-1995. Investigación Económica, 61(237), 25-62. Recuperado de: http://www.jstor.org/stable/42777561

[35] Soares, M. M. y Melo, B. G. D. (2016). Las condiciones políticas y técnicas de las transferencias voluntarias de la Unión a los municipios brasileños. Revista de Administração Pública, 50(4), 539-562. doi:10.1590/00347612138727.

[36] Sobarzo, H. (1999). La cuestión fiscal y el nuevo federalismo. Estudios Sociológicos, 27(51), 743-785. Recuperado de: https://estudiossociologicos.colmex.mx/index.php/es/article/view/611/611

[37] _ (2006). Esfuerzo y potencialidad fiscal de los gobiernos estatales en México: Un sistema fiscal representativo. Ël Trimestre Económico,73(292), 809-861. Recuperado de: http://www.jstor.org/stable/20868353

[38] Sour, L. (2004) El sistema de transferencias federales en México: Premio o castigo para el esfuerzo fiscal de los gobiernos locales urbanos. Gestión y Política Pública, 13(3), 733-753. Recuperado de: https://mpra.ub. uni-muenchen.de/50444/1/MPRA_paper_50444.pdf 
[39] _ (2007). El efecto flypaper de las transferencias intergubernamentales del ramo $\overline{2} 8$ en los gobiernos locales mexicanos, 1990-2004. Recuperado de: http://repositoriodigital.cide.edu/bitstream/handle/11651/813/83544.pdf?sequence=1isAllowed=y

[40] _ (2008). Un repaso de los conceptos sobre capacidad y esfuerzo fiscal, y su aplicación en los gobiernos locales mexicanos. Estudios Demográficos y Urbanos, 23(2), 271-297. doi:10.24201/edu.v23i2.1312

[41] _ (2013). Theflypapereffect in Mexican local governments. Estudios Económicos,28(1), 165-186. Recuperado de: http://www.jstor.org/stable/23609654

[42] Stein, E. (1998). Fiscal decentralization and government size in Latin American. Journal of Applied Economics, 2(2), 357-391. doi: 10.2139/ssrn.1815995.

[43] Toro, S. y Lara, B. (2019). Tactical distribution in local funding: The value of an aligned mayor. European Journal of Political Economy, 56, 74-89. doi:10.1016/j.ejpoleco.2018.07.006

[44] Trujillo, L. (2008). Transferencias intergubernamentales y gasto local: Repensando la descentralización fiscal desde una revisión de la literatura. Gestión y Política Pública, 17(2), 451-486. Recuperado de: http://www.scielo.org.mx/pdf/gpp/v17n2/v17n2a6.pdf

[45] Valenzuela Reynaga, R. e Hinojosa Cruz, A. (2017) Las transferencias federales, los contrapesos políticos y los ingresos fiscales estatales en México. Economía UNAM 14(42), septiembre-diciembre. doi:10.1016/j.eunam.2017.09.002 